\title{
QM/MM: what have we learned, where are we, and where do we go from here?
}

Received: 25 May 2005 / Accepted: 6 October 2005 / Published online: 8 July 2006

(C) Springer-Verlag 2006

\begin{abstract}
This paper briefly reviews the current status of the most popular methods for combined quantum mechani$\mathrm{cal} /$ molecular mechanical (QM/MM) calculations, including their advantages and disadvantages. There is a special emphasis on very general link-atom methods and various ways to treat the charge near the boundary. Mechanical and electric embedding are contrasted. We consider methods applicable to gas-phase organic chemistry, liquid-phase organic and organometallic chemistry, biochemistry, and solid-state chemistry. Then we review some recent tests of QM/MM methods and summarize what we learn about QM/MM from these studies. We also discuss some available software. Finally, we present a few comments about future directions of research in this exciting area, where we focus on more intimate blends of QM with MM.
\end{abstract}

Keywords Boundary treatment . Combined QM/MM . Electrostatic interactions - Embedding scheme - Link atom . Multi-configuration molecular mechanics . Potential energy surfaces

\section{Introduction}

Despite the increasing computational capability available now, molecular modeling and simulation of large, complex systems at the atomic level remain a challenge to computational chemists. At the same time, there is an increasing

Contribution to the Proceedings of the 10th Electronic Computational Chemistry Conference

H. Lin $(\varangle) \cdot$ D. G. Truhlar $(\varangle)$

Chemistry Department and Supercomputing Institute,

University of Minnesota, 207 Pleasant Street SE,

Minneapolis, MN 55455-0431, USA

E-mail: hai.lin@cudenver.edu

E-mail: truhlar@umn.edu

H. Lin

Chemistry Department, University of Colorado at Denver,

PO Box 173364, Denver, CO 80217, USA interest in nanostructured materials, condensed-phase reactions, and catalytic systems, including designer zeolites and enzymes, and in modeling systems over longer time scales that reveal new mechanistic details. The central problem is: can we efficiently accomplish accurate calculations for large reactive systems over long time scales? As usual, we require advances in modeling potential energy surfaces, in statistical mechanical sampling, and in dynamics. The present article is concerned with the potentials.

Models based on classical mechanical constructs such as molecular mechanical (MM) force fields that are based on empirical potentials describing small-amplitude vibrations, torsions, van der Waals interactions, and electrostatic interactions have been widely used in molecular dynamics (MD) simulations of large and complex organic and biological systems [1-25] as well as inorganic and solid-state systems [26-31]. However, the MM force fields are unable to describe the changes in the electronic structure of a system undergoing a chemical reaction. Such changes in electronic structure in processes that involve bond-breaking and bond-forming, charge transfer, and/or electronic excitation, require quantum mechanics $(\mathrm{QM})$ for a proper treatment. However, due to the very demanding computational cost, the application of QM is still limited to relatively small systems consisting of up to tens or several hundreds of atoms, or even smaller systems when the highest levels of theory are employed.

Algorithms that combine quantum mechanics and molecular mechanics provide a solution to this problem. These algorithms in principle combine the accuracy of a quantum mechanical description with the low computational cost of molecular mechanics, and they have become popular in the past decades. The incorporation of quantum mechanics into molecular mechanics can be accomplished in various ways, and one of them is the so-called combined quantum mechanical and molecular mechanical (QM/MM) methodology [32151].

A QM/MM method (see Fig. 1) treats a localized region, e.g., the active site and its neighbors in an enzyme (called the primary subsystem, PS), with QM methods and includes the influence of the surroundings (e.g., the protein environment, 


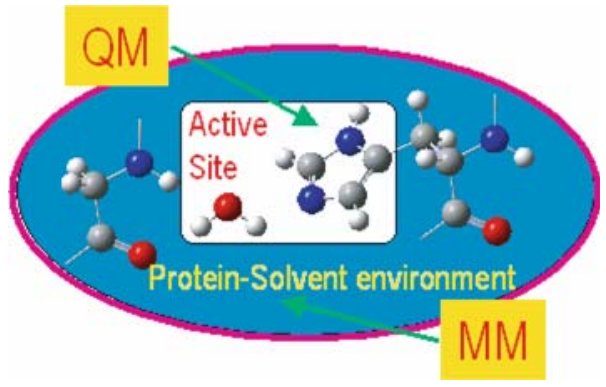

Fig. 1 Illustration for the QM/MM method in the enzyme system. The active center is treated at the QM level and the surroundings is treated at the MM level

called secondary subsystem, or SS) at the MM level. The QM/MM energy for the entire system (ES) can be formally defined by

$$
\begin{aligned}
E(\mathrm{QM} / \mathrm{MM} ; \mathrm{ES})= & E(\mathrm{QM} ; \mathrm{PS})+E(\mathrm{MM} ; \mathrm{SS}) \\
& +E(\mathrm{QM} / \mathrm{MM} ; \mathrm{PS} \mid \mathrm{SS})
\end{aligned}
$$

i.e., as a summation of the energy of the PS, the energy for the SS, and the interaction energy between them. The relation between ES, PS, and SS is given by

$\mathrm{ES}=\mathrm{SS}+\mathrm{PS}$.

The PS is also called the QM subsystem (or - sometimes the MO subsystem), and the SS is often called the MM subsystem. The inclusion of the interactions between the active center and its environment allows a more realistic description of the system, in comparison with isolated QM calculations on subsystems, which are often called model systems. Such a PS-SS coupling is the heart of a QM/MM method.

Recently, there has been much exciting progress on QM/ MM algorithm development, implementation in programs, and applications. In this contribution, we will briefly review the current status of the most popular methods for QM/MM calculations, including their advantages and disadvantages. There are many reviews of QM/MM methods available in literature $[49,58,59,65,66,74,75,81,95,113,119]$. The present study will put a special emphasis on very general link atom methods and various ways to treat the charge near the boundary. Mechanical and electrostatic embedding will be contrasted. We will review some recent tests and applications from our work and that of other groups and summarize what we learn about QM/MM from these studies. We will also discuss some available software. Finally, we will present a few comments about future directions of research in this exciting area. The applications of QM/MM methods are very interesting and very important, but they are not emphasized in this review.

\section{Interactions between the primary and secondary subsystems}

The coupling between the primary system (PS) and the secondary subsystem (SS) is the heart of a QM/MM method. The coupling, in general, must be capable of treating bothbonded (bond stretching, bond bending, and internal rotation, sometimes called valence forces) and non-bonded interactions (electrostatic interaction and van der Waals interactions). Various QM/MM schemes have been developed to treat the interactions between the PS and SS.

As might be expected from its general importance in a myriad of contexts [152], the electrostatic interaction is the key element of the coupling. Depending on the treatment of the electrostatic interaction between the PS and SS, the $\mathrm{QM} / \mathrm{MM}$ schemes can be divided into two groups, the group of mechanical embedding and the group of electric embedding. [44] A mechanical embedding (ME) scheme performs QM computations for the PS in the absence of the SS, and treats the interactions between the PS and SS at the MM level. These interactions usually include both bonded (stretching, bending, and torsional) and non-bonded (electrostatic and van der Waals) interactions. The original integrated molecular-orbital molecular-mechanics (IMOMM) scheme by Morokuma and coworkers [39,52,62], which is also known as the two-layer ONIOM(MO:MM) method, is an ME scheme.

In an electrostatic embedding (EE) scheme, also called electric embedding, the QM computation for the PS is carried out in the presence of the SS by including terms that describe the electrostatic interaction between the PS and SS as one-electron operators that enter the QM Hamiltonian. Since most popular MM force fields, like CHARMM [18] or OPLSAA [17, 19,20,22,24,25], have developed extensive sets of atomic-centered partial point charges for calculating electrostatic interactions at the MM level, it is usually convenient to represent the SS atoms by atomic-centered partial point charges in the effective QM Hamiltonian. However, more complicated representations involving distributed multipoles have also been attempted [46,89]. The bonded (stretching, bending, and torsional) interactions and non-bonded van der Waals interactions between the PS and SS are retained at the MM level.

A comparison between the ME and EE schemes are presented in Table 1, which will be discussed in detail in Sects. 2.1 and 2.2.

\subsection{Mechanical embedding?}

The key difference between an ME scheme and an EE scheme is how they treat the electrostatic interaction between PS and SS. An ME scheme handles the interaction at the MM level, which is simpler. However, such a treatment has drawbacks. First, the treatment requires an accurate set of MM parameters such as atom-centered point charges for both the PS and SS. It is relatively easier to get such parameters for the SS, and the problem with getting such parameters for the PS, where reactions are taking place, was the central reason for moving from MM to QM in the first place. Since the charge distribution in the PS usually changes as reaction progresses, the error in using a single set of MM parameters could be very serious. The second drawback of an ME scheme is that it ignores the potential perturbation of the electronic structure 
Table 1 A Comparison between the ME and EE Schemes

\begin{tabular}{|c|c|c|}
\hline & $\mathrm{ME}$ & $\mathrm{EE}$ \\
\hline $\begin{array}{l}\text { Electrostatic interaction } \\
\text { between the primary system (PS) } \\
\text { and the secondary system (SS) }\end{array}$ & $\begin{array}{l}\text { Handled in the } \\
\text { standard MM way }\end{array}$ & $\begin{array}{l}\text { Treated by including } \\
\text { certain one-electron terms } \\
\text { in the QM Hamiltonian }\end{array}$ \\
\hline Advantage & Simple & $\begin{array}{l}\text { 1. Do not need electrostatic } \\
\text { MM parameters for PS atoms, which } \\
\text { may change their character } \\
\text { during the simulation } \\
\text { 2. The electronic structure of } \\
\text { the PS adjusts to the charge } \\
\text { distribution in the SS }\end{array}$ \\
\hline Disadvantage & $\begin{array}{l}\text { 1. An accurate set of electrostatic } \\
\text { MM parameters is often not available for PS atoms } \\
\text { 2. Ignores the potential perturbation of the } \\
\text { electronic structure of the PS by the } \\
\text { charge distribution of the SS }\end{array}$ & $\begin{array}{l}\text { 1. More computational effort } \\
\text { 2. Need to construct an appropriate } \\
\text { representation for the } \\
\text { charge distribution in the SS }\end{array}$ \\
\hline
\end{tabular}

of the PS due to the electrostatic interaction between the PS and SS. The atom-centered charges in the SS polarize the PS and alter its charge distribution. This is especially a problem if the reaction in the PS is accompanied by charge transfer. Another problematic situation would be a system (e.g., an open-shell system containing transition metals) having several electronic states close in energy, for which the polarization could change the energetic order of these states, e.g., predicting a different ground state with a different charge and/or spin distribution.

To deal with the lack of accurate MM electrostatic parameters for the PS atoms during a reaction, one might consider obtaining these parameters dynamically as the reaction progresses, e.g., deriving atom-centered point charges for the PS atoms when the system evolutes along the reaction path. This idea works in principle, but in practice, it requires a large PS to achieve the desired accuracy due to the second drawback of ME schemes, which was just discussed above. That is, the PS system must be large enough to assure that its calculated charge distribution is converged with respect to the location of the QM/MM boundary. Moreover, an accurate and fast algorithm is necessary to derive the MM electrostatic parameters on the fly (with no or only a little calibration by experimental data or validation by doing pure MM simulation). These requirements will apparently increase the computational effort considerably.

This problem motivates consideration of the mechanically embedded three-layer ONIOM (MO:MO:MM) method [52]. This method attempts to overcome the drawbacks of a mechanically embedded two-layer ONIOM (MO:MM) [39] by introducing a buffer (middle) layer, which is treated by an appropriate lower-level QM theory (e.g., semi-empirical molecular orbital theory), which is computationally less expensive than the method used for the innermost primary subsystem. One can label such a treatment as QM1:QM2:MM or QM1/QM2/MM. The second QM layer is designed to allow a consistent treatment of the polarization of the active center by the environment. The new treatment does improve the description, but, with mechanical embedding, it does not solve the problem completely, since the QM calculation for the first layer is still performed in the absence of the rest of the atoms.

\subsection{Electrostatic embedding?}

In contrast to an ME scheme, an EE scheme does not require the MM electrostatic parameters for the PS atoms because the electrostatic interaction between PS and SS is now treated at a more advanced level by including certain one-electron terms in the QM Hamiltonian. The polarization of the PS by the charge distribution of the SS is also taken into account automatically. The recent progress in the development of electrostatic embedded ONIOM method [137,138] reflects the trend of moving from ME to EE in QM/MM methodology. The price to pay for this improvement is more complicated implementation and increased computational cost.

The unsolved issue for EE schemes is how to construct the one-electron terms in the effective QM Hamiltonian. As mentioned earlier, the simplest way is to represent the charge distribution of the SS as a background of atom-centered partial charges. This is further facilitated by the availability of a set of pre-parameterized MM point charges in many MM force fields; these MM point charges have in principle been parameterized consistently with the other MM parameters to give accurate MM energies, and they have been validated by extensive test calculations. The use of these MM atomcentered partial charges is very efficient, and it is the most popular way in constructing the effective QM Hamiltonian. Nevertheless, the question is raised: are charge parameterized for a particular MM context also appropriate for use in a QM Hamiltonian? In an extreme case, for example, a zeolite-substrate system, the formal atomic charges used in aluminosilicate force field are chosen to reproduce the structural rather than electrostatic data; such charges may not be appropriate for the construction of the one-electron terms in the effective QM Hamiltonian [56].

The MM point charges actually include the contributions due to higher-order multipoles implicitly, i.e., the higherorder contributions are folded into the zero-order parameters. 
By considering higher-order multipole contributions explicitly, one might increase the accuracy of calculated electrostatic interactions, but this makes the implementation more difficult, and the computational costs grow. The development of distributed multipole parameters is also a difficult and time-consuming task, but the biggest obstacle is that the higher-order terms are generally sensitive to the geometry or conformation changes [153-155]. The high conformation dependence of the multipole expansion limits the transferability [156]. For example, only about 20 amino acids are commonly encountered in proteins. It would be ideal to have one set of parameters for these 20 amino acids, which could be used to simulate any proteins, and it would be very inconvenient if one had to develop a new set of parameters whenever another protein is studied or whenever the conformation of a given protein changes considerably.

Another unsolved issue in ascertaining the best EE strategy is the question of the polarization of the SS. In principle, the PS and SS will polarize each other until their charge distributions are self-consistent; properly account for this in a computation is usually accomplished by an iterative scheme [157] (or matrix inversion) or by an extended Lagrangian scheme [158]. Ideally, an EE scheme should include this self-consistency, but usually the charge distribution of the SS is considered frozen for a given set of SS nuclear coordinates. Schemes that relax this constraint can be called self-consistent embedding schemes (or polarized embedding schemes). However, self-consistency is difficult to achieve because it requires a polarizable MM force field [157-169], which has the flexibility to respond to perturbation by an external electric field. Such flexibility is not available in today's most popular MM force fields, although research to develop a polarizable force field has received much attention $[164,166]$. Moreover, the use of a self-consistent embedding scheme also brings additional complication to the treatment of the boundary between the PS and SS, which we will discuss later in next section. Finally, it increases the computational effort, since iterations are required to achieve self-consistent polarization of the PS and SS. Thus, in most EE implementations, the PS is polarized by the SS, but the SS is not polarized by the PS. Early examinations on the self-consistent embedding scheme was carried out by Thompson and Schenter [42] and Bakowies and Thiel [44]. Their treatments are based on models that describe the mutual polarization of QM and MM fragments in the sprit of reaction field [170-173] theory, with the difference that the response is generated by a discrete reaction field (atomic polarizabilities) rather than a continuum. Their results suggests that the polarization of the SS by the PS can be crucial in applications involving a charged PS that generates large electric fields.

\subsection{Interactions other than electrostatic}

Although, as discussed above, the key difference between the $\mathrm{ME}$ and EE schemes is the treatment of the electrostatic inter- action between the PS and the SS, there are also important issues involving in the treatments of the other interactions between the PS and the SS. These interactions include the bonded (stretching, bending, and torsional) interactions and the non-bonded van der Waals interactions, which are handled at the MM level.

A similar question arises here, as in the case of electrostatic interactions for the ME scheme, but now even for the EE schemes, i.e., all the interactions calculated at the MM level rely on the availability of MM parameters for the PS atoms. These parameters are not necessarily the same for the PS atoms in the reactant and product because the atom types are changed for some atoms, e.g., a carbon atom may change from $\mathrm{C}=\mathrm{O}$ type to $\mathrm{C}-\mathrm{O}-\mathrm{H}$ type. Which set of MM parameters should we use? Should one switch between two sets of MM parameters during a dynamics calculation following the reaction path? Switching between these two sets of parameters during a dynamics calculation or along the reaction path is not convenient, and, again, avoiding this was the one of the reasons for moving up from MM to QM. Moreover, even if the switching between parameters could be done, one does not know at which point along the reaction path it should be done and how suddenly if the change is gradual. There is no unambiguous answer.

One key difference between the need for non-bonded electrostatic parameters and the need for bonded parameters is that the latter requirement can always be obviated by making the PS bigger, i.e., moving the QM-MM boundary out. The change of atom types might change the force constants for associated bonded interactions. Usually force constants for stretches are much bigger than force constants for bends, and force constants for torsions are the smallest. The changes of force constants due to the change of atom types are often in this order, too. This provides us with gauge for monitoring the error due to using a single set of MM parameters. The bonded interactions between PS and SS are localized at the boundary. In principle, the use of a larger PS pushes the boundary away from the reaction center and helps to alleviate the uncertainty due to parameter choices, but at a price of increasing computational effort. In many cases, though, enlarging the PS is not a practical solution. What then? Our suggestion is to keep using one set of MM parameters, and examine whether the errors introduced by using one set of parameters exceeds the errors produced by other approximations that are introduced by the QM/MM framework. Although our treatment is not a perfect solution, it is very practical, and it appears to be reasonable.

For the van der Waals interactions, any PS atoms that change atom types are intrinsically ambiguous; this problem cannot be avoided even if a larger QM subsystem is adopted. Fortunately, in practice, it does not appear to be a serious problem in most cases, since the van der Waals interactions are significant only at short distances (as compared to longer range forces associated charged species and permanent dipoles), and the use of only one set of van der Waals parameters is often adequate. 


\subsection{Treating solid-state systems}

So far we have been talking about QM/MM methodology in a very general sense. In this subsection, we more specifically address some question about how to treat periodic systems and other solid-state materials such as metals, metal oxides, and surface-adsorbate systems. Excellent discussions [47,56, $74,85,96,97,101-103,108,115,133,140,145]$ are available for many aspects, and we focus here especially on studies of zeolites.

As we mentioned above, the most important interaction between the PS and the SS is the electrostatic interaction. Thus, the central problem in treating periodic systems like the zeolite-substrate systems is how to incorporate the longrange electrostatic interactions between the SS and PS into a cluster model. The basic idea [174] is to develop a representation of charge distribution with a finite number of multipoles (usually point charges) to mimic the infinite and periodic charge distribution of the environment in which the cluster model is embedded. This effective charge-distribution can be obtained by minimizing the difference between the electrostatic potentials that are generated by the effective charge distribution and by the original infinite and periodic charge distribution at a set of sampling points at the active site. Additional effective core potentials can be associated with selected point charges if needed. For example, parameterized effective core potentials can be used to replace point charges that are close to anions in the PS in order to reduce the overpolarization of these anions [175]. By doing so, one truncates the infinite and periodic system to a finite embedded cluster model, which is now much easier to handle.

A simple example is the surface charge representation of the electrostatic embedding potential (SCREEP) method, in which the electrostatic potential from the infinite crystal lattice is modeled by a finite number (usually several hundreds) of point charges located on a surface enclosing the cluster [176]. More sophisticated models [97,101,103] also include polarization effects on the SS by using the shellmodel [159]. The shell model [159] represents an ion, e.g., an $\mathrm{O}^{2-}$ ion in silica, by a pair of charges, namely, a positive core and a negative shell. The pair of charges are connected by a harmonic potential. The positions of all charge are optimized to get the lowest energy, i.e., the polarization effect is modeled as charge redistribution.

It is a concern that, in QM/MM calculations, as a consequence of the finite size of the cluster, the calculated HOMOLUMO gap for solid is still typically larger than that for the corresponding extended solid, despite corrections to the energy to take into account the electrostatic contribution of the MM region. One might expect this to cause some errors in the calculation of absorption (of ions, electrons, or molecules) into the QM center. One important question that seems to be involved is whether the neglect of orbital interactions between the QM and MM subsystems underestimates the bandwidth of the QM system. This would be a serious problem if the QM-MM boundary passed through a conjugated system or a metallic region. But if the boundary passes through a covalent bond? First, it is important to keep in mind that the HOMO-LUMO gap is not a physical observable, and the LUMO itself is somewhat arbitrary as long as it remains unoccupied. (For example, the LUMO of Hartree-Fock theory is unphysical, and the meaning of orbital energies in DFT is still a subject of debate.) It is most profitable to cast the problem in terms of observables.

An example of a physical observable of concern would be the absorption energy of an electron into the QM region, i.e., the electron affinity of a molecule in the $\mathrm{QM}$ region. This is a difficult question to address because one of the main failings of QM/MM methods is that they neglect charge transfer between the QM and MM subsystems, although in reality there is almost always some charge transfer between nonidentical systems in close proximity, and it is not expected to an integer. Nevertheless we can imagine the case of transferring an integer charge into the QM region and ask whether the electron affinity might be systematically in error, due to a systematic error in the HOMO-LUMO gap caused by neglecting the overlap of QM orbitals with the (missing) MM orbitals. This would be hard to answer because the electron affinity of a subsystem is not well defined. Therefore, one might ask a related practical question such as whether one systematically underestimates the energy of anionic QM subsystems, such as carboxylates. In practice, we have not seen such an effect. The errors due to the inexact treatment of the electrostatic effects of the MM system are large enough that the error in energies of reaction can be in either direction.

Another practical example might be the calculation of electronic excitation energies. Is there a way, other than increasing the size of the QM region, to stabilize the excitation energy? Or: can one calculate accurate electronic excitation energies of a non-isolated QM system without converging the calculation with respect to enlarging the size of subsystem that is treated quantum mechanically. We think that it is reasonable to hope that one can do this, if one makes the QM/MM treatment sophisticated enough. For example, one can obtain reasonable values for solvatochromic shifts from continuum solvation models in which the solvent is not treated quantum mechanically [177].

\subsection{Adaptive QM/MM}

An important issue that arises in simulating liquid-state phenomena and diffusion through solids is the adaptive movement of the quantum mechanical region, which is called the "hot spot"[50,77,116,178]. Algorithms have been reported for liquid-phase simulation that allows water molecules to enter and leave the QM region dynamically. The basic idea is to identify a narrow "buffer region" or "switching shell" between the QM and MM regions. The cut-off is group-based, i.e., a solvent molecule like water is considered to be in the buffer region when its center of mass is in the buffer region. In order to avoid a discontinuity in the force as a solvent molecule enters or leaves the hot spot, Rode and coworkers [50] proposed to use a smooth function for the forces experienced 
by the atoms in the buffer region to ensure a smooth transition between QM and MM force. The smooth function takes the same form as the one [179] used in the CHARMM program to handle the discontinuity in energy and force due to the use of cut-offs for nonbonded (especially electrostatic) interactions. Despite its success, this treatment lacks a unique definition for the energy, which is obtained by integration of the force. Later, Kerdcharoen and Morokuma [116] described another scheme to cope with the discontinuity. In their scheme, two QM/MM calculations are performed for a given configuration of the whole system. The first calculation is done with the atoms in the buffer region and the atoms in the MM region treated at the MM level, and the second calculation is carried out with the atoms in the buffer region and the atoms in the $\mathrm{QM}$ region treated at the $\mathrm{QM}$ level. The total $\mathrm{QM} / \mathrm{MM}$ energy is a weighted average of the $\mathrm{QM} / \mathrm{MM}$ energies obtained in these two calculations; the weight function is determined by the position of the atoms in the buffer region. This treatment can be viewed as making a smooth connection of two potential energy surfaces.

\section{QM/MM boundary treatment}

In this section, we examine the problem with a stronger microscope, and we consider details, especially for the troublesome implementation of the EE scheme. In some cases, the boundary between PS and SS does not go through a covalent bound, e.g., a molecule being solvated in water, where the solute is the PS and the solvent (water) molecules are the SS [36,69]. The effective fragment potential method [46] can also be considered as a special case of MM in this catalog. In many cases, however, one cannot avoid passing the boundary between the PS and SS through covalent bonds (e.g., in enzymes or reactive polymers) or through ionic bonds (in solid-state catalysts). This is called cutting a bond. In such cases, special care is required to treat the boundary, and this section (Sect. 3) is mainly concerned with this problem.

\subsection{Link atom or local orbital?}

Treatments of the boundary between PS and SS regions can be largely grouped into two classes. The first is the so-called link atom approach, where a link atom is used to saturate the dangling bond at the "frontier atom" of the PS. This link atom is usually taken to be a hydrogen atom, $[34,39,52,72,106$, $116,119]$ or a parameterized atom, e.g., a one-free-valence atom in the "connection atom" [70], "pseudobond" [82], and "quantum capping potential" [111] schemes, which involve a parameterized semiempirical Hamiltonian [70] or a parameterized effective core potential (ECP) $[82,111]$ adjusted to mimic the properties of the original bond being cut. The second class of QM/MM methods consists of methods that use localized orbitals at the boundary between the PS and SS. An example is the so-called local self-consistent field (LSCF) algorithm $[35,38,43,51,112]$, where the bonds connecting the PS and SS are represented by a set of strictly localized bond orbitals (SLBOs) that are determined by calculations on small model compounds and assumed to be transferable. The SLBOs are excluded from the self-consistent field (SCF) optimization of the large molecule to prevent their admixture with other QM basis functions. Another approach in the spirit of the LSCF method is the generalized hybrid orbital (GHO) method [63,83,113,123,125,142,144,149]. In this approach, a set of four $s p^{3}$ hybrid orbitals is assigned to each MM boundary atom. The hybridization scheme is determined by the local geometry of the three MM atoms to which the boundary atom is bonded, and the parametrization is assumed to be transferable. The hybrid orbital that is directed toward the frontier QM atom is called the active orbital, and the other three hybrid orbitals are called auxiliary orbitals. All four hybrid orbitals are included in the QM calculations, but the active hybrid orbital participates in the SCF optimizations, while the auxiliary orbitals do not.

Each kind of boundary treatment has its strength and weakness. The link atom method is straightforward and is widely used. However, it introduces the artificial link atoms that are not present in the original molecular system, and this makes the definition of the QM/MM energy more complicated. It also presents complications in optimizations of geometries. In addition, it is found, at least in the original versions of the link atom method that the polarization of the bond between the QM frontier atom and the link atom is unphysical due to the nearby point charge on the MM "boundary atom" (an MM boundary atom is the atom whose bond to a frontier QM atom is cut). The distance between the link atom and the MM boundary atom is about $0.5 \AA$ in the case of cutting a $\mathrm{C}-\mathrm{C}$ bond (the bond distance is about $1.1 \AA$ for a $\mathrm{C}-\mathrm{H}$ bond and about $1.5 \AA$ and for a $\mathrm{C}-\mathrm{C}$ bond). Similar problem is found in the case of cutting a $\mathrm{Si}-\mathrm{O}$ bond (the bond distance is about $1.4 \AA$ for a $\mathrm{Si}-\mathrm{H}$ bond and about $1.6 \AA$ and for a $\mathrm{Si}-\mathrm{O}$ bond). At such a short distance, the validity of using a point charge to represent the distribution of electron density is questionable. Special treatments are applied to the MM charges near the boundary so as to avoid this unphysical polarization $[33,44,70,71,82,93,110,124]$. We will discuss this problem in more detail later in Sect.3.2.

The methods using local orbitals are theoretically more fundamental than the methods using link atoms, since they provide a quantum mechanical description of the charge distribution around the QM/MM boundary. The delocalized representation of charges in these orbitals helps to prevent or reduce the overpolarization that, as mentioned above, is sometimes found in the link-atom methods. However, the local orbital methods are much more complicated than the link atom methods. The local orbital method can be regarded as a mixture of molecular-orbital and valence-bond calculations; a major issue in these studies is the implementation of orthogonality constraints of MOs [142]. Moreover, additional work is required to obtain an accurate representation of the local orbitals before the actual start of a QM/MM calculation. For example, in the LSCF method, the SLBOs are predetermined by calculations on small model compounds, 
and specific force field parameters are needed to be developed to work with the SLBOs. In the GHO method, extensive parameterization for integral scaling factors in the QM calculations is needed $[63,125,142,144,149]$. Such parameters usually require reconsideration if one switches MM scheme (e.g., from CHARMM to OPLS-AA), QM scheme (e.g., from semiempirical molecular orbital methods to density functional theory or post-Hartree-Fock ab initio methods), or QM basis set. The low transferability limits the wide application of the local orbital methods.

The performance of both the link-atom and local-orbital approaches has been examined by extensive test calculations. The conclusion is that reasonably good accuracy can be achieved by both approaches if they are used with special care. It is expected that the development and application of both the link-atom and local-orbital methods will continue in the future.

\subsection{Using link-atom methods}

A central objective in the development of a universal QM/MM algorithm is to make the algorithm as general as possible and to avoid or to minimize the requirement of introducing any new parameters. Thus, for example, one way to define a universally applicable method would be that, when one makes an application to a new system, no MM parameters need to be changed, no QM integral scaling factors needed to be determined, no effective core potentials (ECP) needed to be developed. From this point of view, the link-atom method seems very attractive. Furthermore the method will be more easily built into a standard QM code if the link atom is an ordinary hydrogen atom with a standard basis set. Methods having these features will be examined in more detail in this section.

To facilitate our further discussion, we will label the atoms according to "tiers". The MM boundary atom will be denoted as M1. Those MM atoms directly bonded to M1 will be called second-tier molecular mechanics atoms or M2; similarly, one defines M3 atoms as those MM atoms bonded to M2 atoms .... The QM boundary atom that is directly connected to M1 is labeled as Q1. Similarly, one defines Q2 and $\mathrm{Q} 3$ atoms in the QM subsystem. We will denote the link-atom as HL, which stands for "hydrogen-link", emphasizing that an ordinary hydrogen atom is used/preferred.

\subsubsection{Location of the link-atom}

As we mentioned in the previous section, the link-atom method has its problems. The first problem is the introduction of the coordinates of the link atom, which are extra degrees of freedom. By definition, a link atom is neither a QM nor an $\mathrm{MM}$ atom because it is not present in the original PS or SS. This causes ambiguity to the definition of QM/MM energy for the ES. One way to avoid this problem is to make the coordinates of a link atom depend on the coordinates of the PS frontier atom and the SS boundary atom, i.e., the Q1 and
M1 atoms. Such a constraint removes the extra degrees of freedom due to the link atom. Usually the link-atom is put on the line that connects the corresponding Q1 and M1 atoms. Morokuma and coworkers $[72,180]$ proposed to scaled the $\mathrm{Q} 1-\mathrm{HL}$ distance $R(\mathrm{Q} 1-\mathrm{HL})$ with respect to the $\mathrm{Q} 1-\mathrm{M} 1$ distance $R(\mathrm{Q} 1-\mathrm{M} 1)$ by a scaling factor $C_{\mathrm{HL}}$ :

$R(\mathrm{Q} 1-\mathrm{HL})=C_{\mathrm{HL}} R(\mathrm{Q} 1-\mathrm{M} 1)$

During a QM/MM geometry optimization or a molecular dynamics of reaction path calculation, the equilibrium Q1-HL and Q1-M1 distances are constrained to satisfy Eq. (3). The scaling factor, $C_{H L}$, depends on the nature of the bonds being cut and constructed. It has been suggested [72] that it should be the ratio of standard bond lengths between Q1-HL and Q1-M1 bonds, which is close to 0.71 for replacement of a $\mathrm{C}-\mathrm{C}$ single bond by a $\mathrm{C}-\mathrm{H}$ bond. This treatment is reasonable, and its simplicity facilitates the implementation of analytic energy derivatives (gradient and Hessians). However, the meaning of "standard bond length" is ambiguous. Our treatment is to set the scaling factor by

$C_{\mathrm{HL}}=R_{0}(\mathrm{Q} 1-\mathrm{H}) / R_{0}(\mathrm{Q} 1-\mathrm{M} 1)$,

where $R_{0}(\mathrm{Q} 1-\mathrm{H})$ and $R_{0}(\mathrm{Q} 1-\mathrm{M} 1)$ are the $\mathrm{MM}$ bond distance parameters for the Q1-H and Q1-M1 stretches in the employed MM force field, respectively.

It is worthwhile to mention that Eichinge et al. [73] also proposed a scaled-bond-distance scheme that is similar to the above scheme by Morokuma and coworkers. However, the scheme by Eichinge et al. [73] makes the scaling factor depend on the force constants of the $\mathrm{C}-\mathrm{C}$ stretch and $\mathrm{C}-\mathrm{H}$ stretch instead of the bond distances, and it introduces some additional terms to correct the energy.

\subsubsection{MM charges near the boundary}

Another problem (in fact, the problem that has caused the most concern) for the link atom method, as we mention in the previous section, is the overpolarization of the Q1-HL bond due to the nearby M1 point charge. The main reason for this problem is that at such a short distance (usually about $0.5 \AA$ in the case of cutting a C-C bond and about $0.2 \AA$ in the case of cutting a $\mathrm{Si}-\mathrm{O}$ bond), a point charge assigned to the M1 nucleus does not provide a good approximation for the smeared distribution of charge density. For nearby charge distributions, one must considers screening and charge penetration, and dipole or higher-order multipole moments can also become important. Various approaches have been attempted to avoid or minimize this overpolarization effect, and they are outlined below.

If a scheme does nothing to modify the MM charges, we label the scheme as straight-electrostatic-embedding (SEE). The SEE scheme causes the overpolarization problem.

The simplest way to avoid overpolarization is to ignore the M1 charge by setting it to zero [181]; we call this method the Z1 scheme. One can also zero out both M1 and M2 charges; the method can be called Z2. If all M1, M2, and M3 charges are omitted [33], the scheme is called Z3. The 
$\mathrm{Z} 3$ scheme is the default option for electrostatic embedding in ONIOM calculations carried out by the Gaussian03 package [182], but Gaussian03 also allows one to use scaling factors other than zero for M2, M3, and M4 charges (the M1 charge is always set to zero). The scaled-charge schemes are generalizations of the eliminated-charge scheme. Schemes that eliminate or scale MM charges often result in changing the net charge of the SS, e.g., a neutral SS might become partially charged, and generate artifacts in the calculation of energies or spurious long-range forces.

In many force fields such as CHARMM, the neutrality of certain groups is enforced during the parameterization by imposing a constraint that the sum of charges for several neighboring atoms is zero. An improved eliminated-groupcharge scheme [58] takes advantage of this feature by deleting the atomic charges for the whole group that contains the M1 atom. This ensures that the net charge of the SS does not change. It has been found that this scheme is more robust than the $\mathrm{Z} 1, \mathrm{Z} 2$, and $\mathrm{Z} 3$ schemes because it preserves the charge for SS.

A shifted-charge scheme [67] has been developed to work with force fields where the neutral-groups feature is not available. (Of course, the scheme can also be used for force fields having the neutral-groups feature). In this scheme, called the Shift scheme, the M1 charge is shifted evenly onto the M2 atoms that are connected to M1, and an additional pair of point charges is placed in vicinity of the M2 atom in order to compensate for the modification of the M1-M2 dipole by the initial shift.

As pointed out above, the overpolarization effect is largely due to the poor approximation for the distribution of charge density around the M1 atom by a point charge. Therefore, one might think of using a more realistic description for the charge distribution. Recently, it has been proposed [110,124] to use Gaussian charge distributions instead of point charges for selected atoms.

Recently, we [147] developed two new schemes: a redistributed-charge (RC) scheme and a redistributed-charge-anddipole (RCD) scheme, which are based on combining the link-atom treatment and the local-orbital treatment. As indicated in Fig. 2, both schemes use redistributed charges as molecular mechanical mimics of the auxiliary orbitals associated with the MM boundary atom in the GHO theory. In the RC scheme, the M1 charge is distributed evenly onto midpoints of the M1-M2 bonds, i.e., at the nominal centers of the bond charge distributions. The redistributed charge and M2 charges are further modified in the RCD scheme to restore the original M1-M2 bond dipole. The RC and RCD schemes handle the charges in ways that are justified as molecular mechanical analogs to the quantal description of the charge distribution offered by GHO theory.

\section{Validation of a QM/MM algorithm}

Validating QM/MM methods by comparing to high-level calculations or experiment is essential, since the use of un-
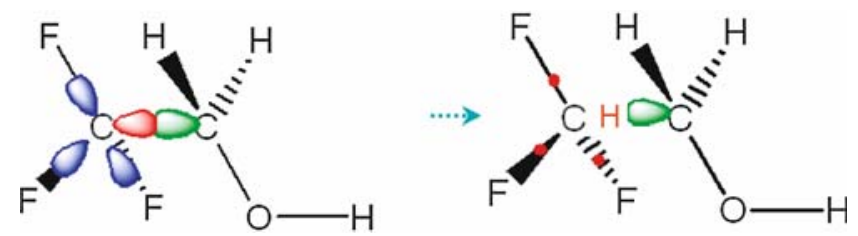

Fig. 2 The redistributed charge scheme (right) is a molecular mechanical analog to the quantal description by the generalized hybrid orbital (GHO) theory (left). The MM boundary atom and the active hybrid orbital (shown in red) in the GHO theory are now modeled by an $\mathrm{H}$ atom, and the auxiliary orbitals (shown in blue) are modeled by three point charges

validated methods is unacceptable. Although the motivation for developing QM/MM methods is to apply them to large systems (e.g., reactions in the condensed phase, including liquids, enzymes, nanoparticles, and solid-state materials), most of the validation studies have been based on small gasphase model systems, where a "model system" is a small- or medium-sized molecule. It is important, in interpreting such validation tests, to keep two important issues in mind.

First, the molecular mechanics parameters, especially partial charges, are usually designed for treating condensedphase systems where partial charges are systematically larger due to polarization effects in the presence of dielectric screening; thus electrostatic effects of the MM subsystem may be overemphasized in the gas phase. Special attention is given to alkyl groups that are frequently involved in these test examples, because a nonpolar $\mathrm{C}-\mathrm{C}$ bond is often considered to be the most suitable place for putting the QM/MM boundary. An alkyl group in the gas phase appears to be very unpolar, and the $\mathrm{C}$ and $\mathrm{H}$ atoms are often assign atom-center point charges of small values. For example, in a recent study [147], charges for the $\mathrm{C}$ and $\mathrm{H}$ atoms in a $\mathrm{C}_{2} \mathrm{H}_{6}$ molecule are $-0.05 \mathrm{e}$ and $0.02 \mathrm{e}$, respectively, as derived by the Merz-Singh-Kollmann $[33,183]$ electrostatic potential (ESP) fitting procedure. An alkyl group becomes more polar in water or in other polar solvents, and the point charges on the atoms in the alkyl group increase significantly. The OPLS-AA force field assigns a charge of $-0.18 \mathrm{e}$ for each $\mathrm{C}$ atom and $0.06 \mathrm{e}$ for each $\mathrm{H}$ atom in the $\mathrm{C}_{2} \mathrm{H}_{6}$ molecule, and in the CHARMM force field, the values are even larger $(-0.27 \mathrm{e}$ for each $\mathrm{C}$ atom and $0.09 \mathrm{e}$ for each $\mathrm{H}$ atom). Our calculations [147] on the proton affinity of several gas-phase molecules having alkyl groups found much bigger errors when using the charges developed for simulations in water than when using the ESP-fitted charges. We believe that our conclusion is general since we tested several QM/MM schemes that treat the MM charges near the boundary differently, and observed a similar trend. We learned from this that it is very hard to test schemes designed for complex processes in the condensed phase by carrying calculations on small molecules in the gas phase.

It is probably more important to focus on the fact that the QM/MM interface can introduce artifacts. Thus, the main goal of validation tests should usually be to ensure that no unacceptably large energetic or structural artifacts are introduced, rather than to achieve high quantitative accuracy for 
MM substituent effects. In this regard, a QM/MM method is often tested by examples that are more difficult in one or another sense than those in a normal application because one wants to know where the performance envelope lies. Thus, calculations on examples having large proton affinities are very suitable for testing. Proton or hydrid transfer involves significant charge transfer and is thus a crucial test for the treatment of electrostatic interactions (especially the procedure for handling MM charges near the QM/MM boundary) in a $\mathrm{QM} / \mathrm{MM}$ method. A large value of the energy difference between the reactant and product also helps us to draw conclusions that are not compromised by the intrinsic uncertainty of the QM/MM approach.

The proton affinity of $\mathrm{CF}_{3} \mathrm{CH}_{2} \mathrm{O}^{-}$, where $\mathrm{CF}_{3}$ is the SS and $\mathrm{CH}_{2} \mathrm{O}^{-}$is the PS, is one of these difficult examples, due to both the close location of the reaction center to the boundary and the presence of significant point charges on the atoms in the $\mathrm{CF}_{3}$ group near the boundary. A recent study [147] on this difficult example by making comparisons between full QM computations and various QM/MM schemes with the ESP-fitted $\mathrm{MM}$ charges for the $\mathrm{CF}_{3}$ group. The QM/MM schemes include the capped PS, the SEE scheme, three eliminated-charge (Z1, Z2, and Z3) schemes, the Shift scheme, the RC scheme, and the RCD scheme. It is found that the Shift and RCD schemes, both of which preserve both the charge of the SS and the M1-M2 bond dipoles, are superior to the other schemes in comparison. For example, when, the errors for the RCD and Shift schemes are 1 and $2 \mathrm{kcal} / \mathrm{mol}$, respectively. It is also found that the largest error is caused by the Z1 scheme $(75 \mathrm{kcal} / \mathrm{mol})$, where neither the charge nor the dipole is preserved. The results suggest that it is critical to retain the feature of charge distribution near the QM/MM boundary. By this criterion, the SEE scheme does not seem to be too bad with an error of $9 \mathrm{kcal} / \mathrm{mol}$; actually it is even better than the $\mathrm{RC}$ scheme (error of $12 \mathrm{kcal} / \mathrm{mol}$ ) and the best charge-elimination schemes Z2 and Z3 (errors of $25 \mathrm{kcal} / \mathrm{mol}$ ). However, the error in the optimized Q1-M1 (C-C) distance is 3-4 times larger for the SEE scheme than for any of the other schemes to which comparison was made, and this makes the SEE scheme a poor choice in practical applications.

\section{Implementation and software}

As summarized in a recent review article, there are basically three kinds of programming architecture for implementing QM/MM methods.

1. Extension of a "traditional" QM package by incorporating the MM environment as a perturbation. Many QM packages have added or are adding the QM/MM options. A well-known example is the ONIOM method implemented in Gaussian03 (http://www.gaussian.com/). Other examples include the ADF(http://www.scm.com/), CHIMISTE/MM (http://www.lctn.uhp-nancy.fr/logiciels. html), GAMESS- UK (http://www.cse.clrc.ac.uk/qcg/ gamess-uk/), MCQUB (http://www.chem.umn.edu/groups/ gao/software.htm), MOLCAS(http://www.teokem.lu.se/ molca s/), MOZYME (http://www.chem.ac.ru/Chemistry/ Soft/ MOZYME.en.html), and QSite (http://www.schrodinger.com/Products/qsite.html) packages.

2. Extension of a "traditional" MM package by incorporating a QM code as a force-field extension. Examples include AMBER (http://www.amber.scripps.edu/), CHARMM (http://www.charmm.org/), and CGPLUS (http://www. comp.chem.umn.edu/cgplus/).

3. A central control program interfacing QM and MM packages, where users can select between several QM and/or MM packages. For example, CHEMSHELL (http://www.cse. clrc.ac.uk/qcg/chemshell/) and QMMM (http://www.comp. chem.umn.edu/qmmm/) belong to this catalog.

Each kind of program architecture has its own merits and disadvantages. The options based on extension of traditional QM and/or MM packages can make use of the many features of the original program, for example, the ability of the MM program to manipulate large, complex biological systems. The disadvantage is that both options need modification of the codes.

The third option is based on module construction and is more flexible. It often needs no or little modification on the original QM and MM programs. The program is automatically updated when the QM or MM packages that it interfaces are updated. The drawback is that it requires a considerable amount of effort to transfer the data between the QM and MM packages, which is usually done by reading data from files, rearranging the data, and writing the data to files. Such additional manipulations can lower the efficiency.

Our recently developed program QMMM adopts the third programming architecture. The QMMMprogram currently interfaces Gaussian03 for doing QM computations and TINKER for doing MM calculations. Geometry optimization and transition-state searching can be done by using the algorithm built into the QMMM program or by using an optimizer in the Gaussian03 program via the external option of Gaussian03. In addition to the RC and RCD schemes, the QMMM program also implements several other schemes such as the SEE, scaled-charge, and shifted-charge schemes for handling the MM point charges near the QM/MM boundary. Currently we are working on combination of the QMMM program with the molecular dynamics program POLYRATE (http://comp.chem. umn.edu/polyrate/) for doing QM/MM direct dynamics calculations [184].

\section{What do we learn from a QM/MM calculation?}

As discussed in the Sect. 1, one benefit that QM/MM calculations bring to us is the inclusion of the effect of a chemical environment (secondary subsystem, SS) on the reaction center (primary subsystem, PS). The interactions between a PS and an SS are of two kinds: (1) interactions that are significant even at long range (electrostatic interaction), and (2) interactions that are local (bonded interactions) or are only significant at short range (van der Waals interactions). The electrostatic interactions are usually dominant, as they 
perturb the electronic structure of the PS, and they often have great effects on energetic quantities such as the reaction energy. The bonded and van der Waals interaction act in other ways; for example in enzyme reactions or solid-state reactions, they impose geometry constraints by providing a rigid frame in the active site or lattice site to hold the PS (in fact, the electrostatic interaction will also affect the equilibrium geometry of the PS).

A practical way to examine the effect of the environment is to compare quantities such as reaction energies or barrier heights as calculated from an isolated QM model and from a QM/MM model. Usually such quantities show significant differences for processes that involve significant changes in the charge distribution. The calculations for proton transfer reactions are good examples (see the discussion on the proton affinity in Sect. 4).

However, one sometimes finds the same or very similar reaction energies and barrier heights from isolated QM model systems and QM/MM model calculations. This is likely to be observed for a reaction without much change transfer, e.g., a radical abstraction reaction. This does not mean that the SS does not affect the PS. In such a case, it is likely that the effect due to the SS is roughly the same for the reactant, transition state, and product, and the cancellation leads to small net effects. An approximate analysis of the effects due to SS can be obtained by an energy-decomposition as follows (see also Fig. 3).

The energy difference between the QM energy for the PS (or CPS, i.e., a PS capped by link atoms) in the gas phase and the QM energy for the PS in an interacting MM environment is defined by

$$
E_{\mathrm{PS} / \mathrm{MM}}=E(\mathrm{QM} ; \mathrm{PS} * *)-E(\mathrm{QM} ; \mathrm{PS}),
$$

where $E(\mathrm{QM} ; \mathrm{PS} * *)$ is the $\mathrm{QM}$ energy for the $\mathrm{PS}$ embedded in the background point charges of $\mathrm{SS}$, and $E(\mathrm{QM} ; \mathrm{PS})$ is the QM energy for the PS in the gas phase. In either case, the geometry is fully optimized at the corresponding level of theory, i.e., at the $\mathrm{QM} / \mathrm{MM}$ level for $E(\mathrm{QM} ; \mathrm{PS} * *)$ and at the $\mathrm{QM}$ level for $E(\mathrm{QM} ; \mathrm{PS})$. Equation (5) provides a measure of the magnitude of the perturbation on the QM subsystem due to the MM subsystem. Generally speaking, the two geometries in Eq. (5) are different. We further decompose $E_{\mathrm{PS} / \mathrm{MM}}$ into two contributions: the energy due to the polarization of the background point charges $\left(E_{\mathrm{pol}}\right)$ and the energy due to the geometry distortion from the PS in the gas phase $\left(E_{\text {steric }}\right)$, which are defined as

$$
\begin{array}{r}
E_{\mathrm{pol}}=E(\mathrm{QM} ; \mathrm{PS} * *)-E\left(\mathrm{QM} ; \mathrm{PS}^{\mathrm{dis}}\right), \\
E_{\text {steric }}=E\left(\mathrm{QM} ; \mathrm{PS}^{\mathrm{dis}}\right)-E(\mathrm{QM} ; \mathrm{PS}), \\
E_{\mathrm{PS} / \mathrm{MM}}=E_{\mathrm{pol}}+E_{\text {steric }},
\end{array}
$$

where $E\left(\mathrm{QM} ; \mathrm{PS}^{\text {dis }}\right)$ is the gas-phase single-point PS energy for the QM/MM optimized geometry, i.e., one takes the PS geometry that resulted from QM/MM optimization and removes the MM point charges. Although such an energydecomposition is approximate, it is informative and provides us deeper understanding of the QM/MM calculations.

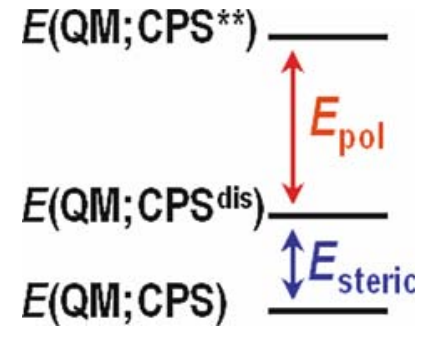

Fig. 3 Decomposition of energy due to MM environment into two contributions: the energy due to the polarization by the background point charges $\left(E_{\mathrm{pol}}\right)$ and the energy due to the geometry distortion $\left(E_{\text {steric }}\right)$. See Eqs. (5), (6), (7), (8)

The energy decomposition is applied to a reaction that we studied recently [147] (Fig. 4).

$$
\mathrm{CH}_{3}+\mathrm{CH}_{3} \mathrm{CH}_{2} \mathrm{CH}_{2} \mathrm{OH} \rightarrow \mathrm{CH}_{4}+\mathrm{CH}_{2} \mathrm{CH}_{2} \mathrm{CH}_{2} \mathrm{OH}
$$

The PS is $\mathrm{CH}_{3}+\mathrm{CH}_{3} \mathrm{CH}_{2}$, giving rise to a CPS as $\mathrm{CH}_{3}+$ $\mathrm{CH}_{3} \mathrm{CH}_{3}$. The $\mathrm{SS}$ is $\mathrm{CH}_{2} \mathrm{OH}$. For each of the reactant, saddle point, and product of this reaction, we found a small steric effect $(0.1 \mathrm{kcal} / \mathrm{mol})$ and a dominant polarization effect $(9 \mathrm{kcal} / \mathrm{mol})$. It is not surprising to see such a small setic effect, since the distortion of geometry for the CPS from the fully relaxed geometry in the gas phase can be rather small. The critical point is that the energies due to geometry distortion and polarization are so similar along the reaction path that they almost cancel out, giving rise to negligibly small net contributions to the reaction energy and barrier height.

Although the MM environment does not have a large net effect on the relative energies of the $\mathrm{H}$ atom transfer reaction (R1), it does have effects on the electronic structure of the CPS through polarization and perturbs the charge distribution. The ESP-fitted charges in Fig. 4 clearly show a trend of stepwise change from the unperturbed CPS (denoted as CPS), to the CPS with distorted geometry (CPS ${ }^{\text {dis }}$ ), then to the CPS embedded in the background point charge distribution (CPS**), and finally to the ES, as modeled by full QM calculations. It is interesting to note that the $\mathrm{Cb}-\mathrm{Cc}$ bond seems to be very unpolar according to the CPS calculations, with a small bond dipole pointing from the $\mathrm{Cb}$ to the $\mathrm{Cc}$ atom. The CPS** calculations predict that the $\mathrm{Cb}-\mathrm{Cc}$ bond is more polar, with a larger and inverse bond dipole pointing from the $\mathrm{Cc}$ to the $\mathrm{Cb}$ atom, in qualitative agreement with full $\mathrm{QM}$ calculations. The CPS** result is generally closer to the full QM results, suggesting that $\mathrm{QM} / \mathrm{MM}$ calculations provide a more realistic description for the QM subsystem than the isolated gas-phase QM model calculations.

The conclusion that for a reaction that does not involve much charge transfer, the inclusion of the electrostatic field of the SS will yield small effects in relative energies but large affects in the electronic structure of the PS also gain supports from studies of zeolite-substrate systems [56]. In [56], it has been found that the inclusion of the electrostatic field of the SS slightly alters the barrier (by ca. $2 \mathrm{kcal} / \mathrm{mol}$ ) of a methyl shift reaction over a zeolite acid site, but has considerably large effects on the charge distribution and vibrational 

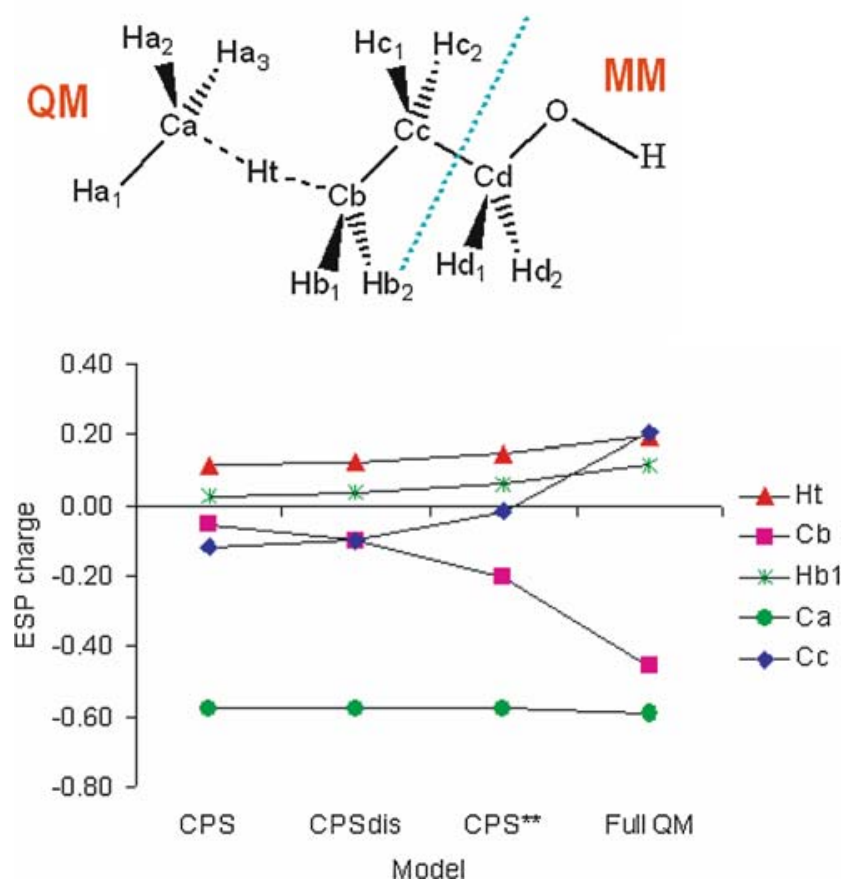

Fig. 4 ESP-fitted charges for selected atoms in reaction $\mathrm{CH}_{3}+$ $\mathrm{CH}_{3} \mathrm{CH}_{2} \mathrm{CH}_{2} \mathrm{OH} \rightarrow \mathrm{CH}_{4}+\mathrm{CH}_{2} \mathrm{CH}_{2} \mathrm{CH}_{2} \mathrm{OH}$

frequencies. For example, the charge on the $\mathrm{O}$ atoms is changed by $0.16 \mathrm{e}$.

Comparing the results of $\mathrm{QM} / \mathrm{MM}$ calculations with experimental results is the ultimate test of a $\mathrm{QM} / \mathrm{MM}$ scheme. However, such a comparison is not trivial, and we mention here several points that need attention in general.

First, one should consider what kinds of experimental results, including their temperature and pressure, are most informative to be compared, and how reliable these results are, i.e., what is the error bar associated with the observed quantity. It is also important to understand, in the case of the experimental data were derived from fitting to a simplified model, what kinds of assumptions and simplifications have been invoked. In addition, it is important to understand the parameters characterizing the QM/MM calculations. For example are the results converged with respect to increasing the size of the PS, increasing the QM level of theory and/or basis sets, tightening of the optimization convergence threshold, and increasing the cutoff distance or cutoff threshold if any, in the calculation of MM nonbonded interactions? If we are treating a complex system like an enzyme solvated in water, is the phase space sampled adequately? If we cannot afford an extensive phase-space sampling, can we examine several representative conformations? Do we need to consider potential anharmonicity in vibrational analysis?

Of particular importance is to separate, at least approximately, the errors due to the insufficient QM treatment of the PS and the errors due to the insufficient consideration of the SS effect. As we showed previously, the barrier height and reaction energy are often not sensitive to the electrostatic interaction between the PS and SS for a reaction that does not involve significant charge transfer. In such a case, it may be more desirable to increase the QM level of theory to improve the results than to improve the MM level or the $\mathrm{QM} / \mathrm{MM}$ interface. On the other hand, for reactions that are sensitive to the electrostatic interaction between the PS and the SS, simply increasing the QM level of theory does not necessarily improve the reliability.

\section{Where do we go from here?}

Combining QM and MM by applying them to separate subsystems with a boundary in physical space is very natural, and it is safe to say that it is now a permanent part of the theoretical toolbox. However, there are other ways to combine QM and MM, and future work may see greater use of methods that blend QM and MM more intimately.

A venerable example of such an approach is the use of quantum mechanics to suggest new functional forms for molecular mechanics. The oldest example would be the Morse curve, which was originally derived by a QM treatment of $\mathrm{H}_{2}^{+}$[185]. Replacing a harmonic bond stretching potential by a Morse curve allows MM to treat bond breaking. It is much harder to treat synchronous bond breaking and bond forming, but this was also accomplished in the early days of quantum mechanics, resulting in the London equation [186-188]. Raff [189] was apparently the first to combine the valence-bond-derived QM London equation with molecular mechanics terms to make a QM/MM reactive potential, and in recent years many other workers, $[190,191]$ especially Espinosa-Garcia and coworkers [192-197], have made fruitful use of this technique. Various workers, of whom we single out Brenner [198-200] and Goddard and coworkers [201-204] for noteworthy systematic efforts, have made generalizations to more complex reactive systems. However, these methods are neither universal nor systematically improvable like the methods discussed in Sect. 3 and 4.

One way to make the combination of valence bond theory and molecular mechanics more universal and systematic is multi-configuration molecular mechanics [205-209] (MCMM). MCMM combines QM and MM in a different way than QM/MM; in MCMM the whole system is treated by $\mathrm{QM}$, and simultaneously the whole system is treated by MM. In fact, every atom is treated by two different MM schemes, one corresponding to a reactant and the other to a product, and the two MM energy expansions interact by a $2 \times 2$ configuration interaction matrix highly reminiscent of London's $2 \times 2$ matrix matrices or the similar $2 \times 2$ matrices used by Raff [189], Warshel and Weiss [210], and others. However, in MCMM the off-diagonal elements of the Hamiltonian are not empirical MM parameters as in previous work, but rather are determined by systematically improvable QM methods. The method is very young but very promising.

In the future, we can expect further progress in MCMM. One straightforward improvement is to use QM/MM to replace QM in determination of the off-diagonal element of the Hamiltonian matrices, and this scheme can be called 
QM/MM-based-MCMM, or QM/MM-MCMM for short. Another even more promising scheme is to combine MCMM with MM in the "same way" that QM is combined with MM in combined $\mathrm{QM} / \mathrm{MM}$ methods. That is, use MCMM to replace the QM in QM/MM; this scheme can be called MCMMbased-QM/MM, or MCMM-QM/MM. Both schemes make MCMM suitable for handling very large systems. Work in this direction is in progress, and encouraging preliminary results have been obtained [211].

Another area of future improvement is use of improved MM formalisms in QM/MM methods. We have already mentioned polarizable MM force fields (see Sect. 2.2) [166]. Even without allowing polarization, the charges in MM force fields can be improved in various ways. In particular, various charge models have been developed or are in refinement. Examples include the restrained electrostatic potential [212] (RESP) fitting procedure and its latest improved version "dynamically generated restrained electrostatic potential" (DRESP) [213] for QM/MM calculations, the family of CM $x(x=1$, [214] 2, [215,216], 3 [217-219], and 4 [220]) charge models, the charge equilibration [221,222] (CEq) method, and the consistent charge equilibration (CQEq) [223-225] method.

An interesting and important future development is the adaptive QM/MM scheme, which was discussed in Sect. 2.5. If we allow atoms to be exchanged between the QM and MM regions, can we take one more step forward, allowing fractional (or whole) charges to be transferred between the $\mathrm{QM}$ and MM regions? Of course, to accomplish this goal, we need to work out how to describe the electronic structure of a system with fractional electrons [226].

Another important trend we can expect to see in the near future is the incorporation in MM of methods for modeling metallic systems that were developed in the physics community. For example, just as simplified valence bond theory can be used to obtain functional forms for extending MM to reacting systems, the second-moment approximation to the tight-binding approximation (also known as extended Hückel theory) can be used to obtain new forms for modeling metals [227]. An example of this kind of approach is the use of the embedded-atom functional form to develop MM potentials for Al nanoparticles [228].

A theme that emerges from several of the approaches discussed in this section is the difficulty of classifying the potential energy function as QM or MM. For example, is the embedded-atom method an approximate version of density functional theory or is it MM? Is MCMM an automatic fitting method for QM energies or is it an extension of MM to reactive systems? We prefer to think of these methods as new kinds of QM-MM hybrids where the QM-MM combination is more intimate than in the first generation of combined QM/MM methods.

Acknowledgements This work is supported in part by the National Science Foundation and the Office of Naval Research. The authors are grateful to Jiali Gao and Jingzhi Pu for valuable discussions.

\section{References}

1. van Gunsteren WF, Berendsen HJC, Geurtsen RG, Zwinderman HRJ (1986) Ann NY Acad Sci 482:287

2. Allinger NL, Yuh YH, Lii JH (1989) J Am Chem Soc 111:8551

3. Lii JH, Allinger NL (1989) J Am Chem Soc 111:8566

4. Lii JH, Allinger NL (1989) J Am Chem Soc 111:8576

5. Mayo SL, Olafson BD, Goddard WA III (1990) J Phys Chem 94:8897

6. Rappé AK, Casewit CJ, Colwell KS, Goddard III WA Skid WM (1992) J Am Chem Soc 114:10024

7. Casewit CJ, Colwell KS, Rappé AK (1992) J Am Chem Soc 114:10035

8. Casewit CJ, Colwell KS, Rappé AK (1992) J Am Chem Soc 114:10046

9. Rappé AK, Colwell KS, Casewit CJ (1993) Inorg Chem 32:3438

10. Cornell WD, Cieplak P, Bayly CI, Gould IR, Merz KM Jr, Ferguson DM, Spellmeyer DC, Fox T, Caldwell JW, Kollman PA (1995) J Am Chem Soc 117:5179

11. Pearlman DA, Case DA, Caldwell JW, Ross WS, Cheatham TEI, DeBolt S, Ferguson D, Seibel G, Kollman PA (1995) Comp Phys Commun 91:1

12. Halgren TA (1996) J Comput Chem 17:490

13. Halgren TA (1996) J Comput Chem 17:520

14. Halgren TA (1996) J Comput Chem 17:552

15. Halgren TA, Nachbar RB (1996) J Comput Chem 17:587

16. Halgren TA (1996) J Comput Chem 17:616

17. Jorgensen WL, Maxwell DS, Tirado-Rives J (1996) J Am Chem Soc 118:11225

18. MacKerell AD Jr, Bashford D, Bellott M, Dunbrack RL, Evanseck JD, Field MJ, Fischer S, Gao J, Guo H, Ha S, Joseph-McCarthy D, Kuchnir L, Kuczera K, Lau FTK, Mattos C, Michnick S, Ngo T, Nguyen DT, Prodhom B, Reiher WE III Roux B, Schlenkrich M, Smith JC, Stote R, Straub J, Watanabe M, Wiorkiewicz-Kuczera J, Yin D, Karplus M (1998) J Phys Chem B 102:3586

19. Jorgensen WL, McDonald NA (1998) Theochem 424:145

20. McDonald NA, Jorgensen WL (1998) J Phys Chem B 102:8049

21. Ewig CS, Thacher TS, Hagler AT (1999) J Phys Chem B 103:6999

22. Rizzo RC, Jorgensen WL (1999) J Am Chem Soc 121:4827

23. Allinger NL, Durkin KA (2000) J Comput Chem 21:1229

24. Kaminski GA, Friesner RA, Tirado-Rives J, Jorgensen WL (2001) J Phys Chem B 105:6474

25. Price MLP, Ostrovsky D, Jorgensen WL (2001) J Comput Chem $22: 1340$

26. Hill J-R, Sauer J (1994) J Phys Chem 98:1238

27. Hill J-R, Sauer J (1995) J Phys Chem 99:9536

28. Schröder K-P, Sauer J (1996) J Phys Chem 100:11043

29. Sierka M, Sauer J (1997) Faraday Discuss 106:41

30. Landis CR, Cleveland T, Firman TK (1998) J Am Chem Soc 120:2641

31. Firman TK, Landis CR (2001) J Am Chem Soc 123:11728

32. Warshel A, Levitt M (1976) J Mol Biol 103:227

33. Singh UC, Kollmann PA (1986) J Comput Chem 7:718

34. Field MJ, Bash PA, Karplus M (1990) J Comput Chem 11:700

35. Ferenczy GG, Rivail J-L, Surjan PR, Naray-Szabo G (1992) J Comput Chem 13:830

36. Gao J, Xia X (1992) Science 258:631

37. Åaqvist J, Warshel A (1993) Chem Rev 93:2523

38. Thery V, Rinaldi D, Rivail J-L, Maigret B, Ferenczy GG (1994) J Comput Chem 15:269

39. Maseras F, Morokuma K (1995) J Comput Chem 16:1170

40. Stanton RV, Hartsough DS, Merz KM Jr, (1995) J Comput Chem $16: 113$

41. Thompson MA (1995) J Phys Chem 99:4794

42. Thompson MA, Schenter GK (1995) J Phys Chem 99:6374

43. Assfeld X, Rivail J-L (1996) Chem Phys Lett 263:100

44. Bakowies D, Thiel W (1996) J Phys Chem 100:10580

45. Bakowies D, Thiel W (1996) J Comput Chem 17:87

46. Day PN, Jensen JH, Gordon MS, Webb SP, Stevens WJ, Krauss M, Garmer D, Basch H, Cohen D (1996) J Chem Phys 105:1968 47. Eichler U, Kölmel CM, Sauer J (1996) J Comput Chem 18:463 
48. Eurenius KP, Chatfield DC, Brooks BR, Hodoscek M (1996) Int J Quantum Chem 60:1189

49. Gao (1996) J Rev Comput Chem 7:119

50. Kerdcharoen T, Liedl KR, Rode BM (1996) Chem Phys 211:313

51. Monard G, Loos M, Thery V, Baka K, Rivail J-L (1996) Int J Quantum Chem 58:153

52. Svensson M, Humbel S, Froese RDJ, Matsubara T, Sieber S, Morokuma K (1996) J Phys Chem 100:19357

53. Bersuker IB, Leong MK, Boggs JE, Pearlman RS (1997) Int J Quantum Chem 63:1051

54. Cummins PL, Gready JE (1997) J Comput Chem 18:1496

55. Gao J, Alhambra C (1997) J Chem Phys 107:1212

56. Sherwood P, De Vries AH, Collins SJ, Greatbanks SP, Burton NA, Vincent MA, Hillier IH, (1997) Faraday Discuss 106:79

57. Tongraar A, Liedl KR, Rode BM (1997) J Phys Chem A 101:6299

58. Antes I, Thiel W (1998) ACS Symp Ser 712:50

59. Bentzien J, Florian J, Glennon TM, Warshel A (1998) ACS Symp Ser 712:16

60. Bersuker IB, Leong MK, Boggs JE, Pearlman RS (1998) ACS Symp Ser 712:66

61. Burton NA, Harrison MJ, Hart JC, Hillier IH, Sheppard DW (1998) Faraday Discuss 110:463

62. Froese RDJ, Musaev DG, Morokuma K (1998) J Am Chem Soc 120:1581

63. Gao J, Amara P, Alhambra C, Field MJ (1998) J Phys Chem A $102: 4714$

64. Kaminski GA, Jorgensen WL (1998) J Phys Chem B 102:1787

65. Merz KM Jr (1998) ACS Symp Ser 712:2

66. Mordasini T, Thiel W (1998) Chimia 52:288

67. Sinclair PE, de Vries A, Sherwood P, Catlow CRA, van Santen RA (1998) J Chem Soc Faraday Trans 94:3401

68. Stefanovich EV, Truong TN (1998) ACS Symp Ser 712:92

69. Woo TK, Cavallo L, Ziegler T (1998) Theor Chem Acc 100:307

70. Antes I, Thiel W (1999) J Phys Chem A 103:9290

71. de Vries AH, Sherwood P, Collins SJ, Rigby AM, Rigutto M, Kramer GJ (1999) J Phys Chem B 103:6133

72. Dapprich S, Komiromi I, Byun KS, Morokuma K, Frisch MJ (1999) Theochem 461-462:1

73. Eichinger M, Tavan P, Hutter J, Parrinello M (1999) J Chem Phys 110:10452

74. Hillier IH (1999) THEOCHEM 463:45

75. Monard G, Merz KM Jr (1999) Acc Chem Res 32:904

76. Lyne PD, Hodoscek M, Karplus M (1999) J Phys Chem A 103:3462

77. Marini GW, Liedl KR, Rode BM (1999) J Phys Chem A 103:11387

78. Philipp DM, Friesner RA (1999) J Comput Chem 20:1468

79. Pitarch J, Pascual-Ahuir JL, Silla E, Tunon I, Ruiz-Lopez MF (1999) J Comput Chem 20:1401

80. Turner AJ, Moliner V, Williams IH (1999) Phys Chem Chem Phys $1: 1323$

81. Woo TK, Margl PM, Deng L, Cavallo L, Ziegler T (1999) ACS Symp Ser 721:173

82. Zhang Y, Lee T-S, Yang W (1999) J Chem Phys 110:46

83. Amara P, Field MJ, Alhambra C, Gao J (2000) Theor Chem Acc 104:336

84. Byun K, Gao J (2000) J Mol Graph Model 18:50

85. Derenzo SE, Klintenberg MK, Weber MJ (2000) J Chem Phys 112:2074

86. Field MJ, Albe M, Bret C, Martin FP-D, Thomas A (2000) J Comput Chem 21:1088

87. Gogonea V, Westerhoff LM, Merz KM Jr (2000) J Chem Phys 113:5604

88. Hall RJ, Hindle SA, Burton NA, Hillier IH (2000) J Comput Chem 21:1433

89. Kairys V, Jensen JH (2000) J Phys Chem A 104:6656

90. Moliner V, Williams IH (2000) Chem Commun 1843

91. Murphy RB, Philipp DM, Friesner RA (2000) J Comput Chem $21: 1442$

92. Murphy RB, Philipp DM, Friesner RA (2000) Chem Phys Lett $321: 113$
93. Reuter N, Dejaegere A, Maigret B, Karplus M (2000) J Phys Chem A 104:1720

94. Röthlisberger U, Carloni P, Doclo K, Parrinello M (2000) J Biol Inorg Chem 5:236

95. Sherwood P (2000) In: Grotendorst J (ed) Modern methods and algorithms of quantum chemistry. NIC-Directors: Princeton, vol 3, pp 285

96. Sierka M, Sauer J (2000) J Chem Phys 112:6983

97. Sushko PV, Shluger AL, Catlow CRA (2000) Surface Science 450:153

98. Vreven T, Morokuma K (2000) J Comput Chem 21:1419

99. Woo TK, Blöchl PE, Ziegler T (2000) Theochem 506:313

100. Cui Q, Elstner M, Kaxiras E, Frauenheim T, Karplus M (2001) J Phys Chem B 105:569

101. French SA, Sokol AA, Bromley ST, Catlow CRA, Rogers SC, King F, Sherwood P (2001) Angew Chem 113:4569

102. Khaliullin RZ, Bell AT, Kazansky VB (2001) J Phys Chem A 105:10454

103. Nasluzov VA, Rivanenkov VV, Gordienko AB, Neyman KM, Birkenheuer U, Rösch N (2001) J Chem Phy 115:8157

104. Nicoll RM, Hindle SA, MacKenzie G, Hillier IH, Burton NA (2001) Theor Chem Acc 106:105

105. Poteau R, Ortega I, Alary F, Solis AR, Barthelat JC, Daudey JP (2001) J Phys Chem A 105:198

106. Vreven T, Mennucci B, da Silva CO, Morokuma K, Tomasi J (2001) J Chem Phys 115:62

107. Worthington SE, Krauss M (2001) J Phys Chem B 105:7096

108. Batista ER, Friesner RA (2002) J Phys Chem B 106:8136

109. Colombo MC, Guidoni L, Laio A, Magistrato A, Maurer P, Piana S, Rohrig U, Spiegel K, Sulpizi M, VandeVondele J, Zumstein M, Röthlisberger U (2002) Chimia 56:13

110. Das D, Eurenius KP, Billings EM, Sherwood P, Chatfield DC, Hodoscek M, Brooks BR (2002) J Chem Phys 117:10534

111. DiLabio GA, Hurley MM, Christiansen PA (2002) J Chem Phys 116:9578

112. Ferré Nicolas, Assfeld X Rivail J-L (2002) J Comput Chem 23:610

113. Gao J, Truhlar DG, (2002) Annu Rev Phys Chem 53:467

114. Gogonea V (2002) Internet Electron J Mol Design 1:173

115. Hermida-Ramón JM, Karlström G, Lindh R (2002) J Phys Chem B 106:7115

116. Kerdcharoen T, Morokuma K (2002) Chem Phys Lett $355: 257$

117. Laio A, VandeVondele J, Röthlisberger U (2002) J Chem Phys 116:6941

118. Lennartz C, Schaefer A, Terstegen F, Thiel W (2002) J Phys Chem B 106:1758

119. Morokuma K (2002) Phil Trans Roy Soc London, Ser A 360:1149

120. Rajamani R, Gao J (2002) J Comput Chem 23:96

121. Schöneboom JC, Lin H, Reuter N, Thiel W, Cohen S, Ogliaro F, Shaik S (2002) J Am Chem Soc 124:8142

122. Titmuss SJ, Cummins PL, Rendell AP, Bliznyuk AA, Gready JE (2002) J Comput Chem 23:1314

123. Truhlar DG, Gao J, Alhambra C, Garcia-Viloca M, Corchado J, Sanchez ML, Villa (2002) J Acc Chem Res 35:341

124. Amara P, Field MJ (2003) Theor Chem Acc 109:43

125. Devi-Kesavan LS, Garcia-Viloca M, Gao J (2003) Theor Chem Acc 109:133

126. Dinner AR, Lopez X, Karplus M (2003) Theor Chem Acc 109:118

127. Hu H, Elstner M, Hermans J (2003) Proteins 50:451

128. Kongsted J, Osted A, Mikkelsen KV, Christiansen O (2003) J Phys Chem A 107:2578

129. Li G, Zhang X, Cui Q (2003) J Phys Chem B 107:8643

130. Loferer MJ, Loeffler HH, Liedl KR (2003) J Comput Chem 24:1240

131. Molina PA, Sikorski RS, Jensen JH (2003) Theor Chem Acc 109:100

132. Mordasini T, Curioni A, Andreoni W (2003) J Biol Chem 278:4381

133. Sherwood P, de Vries AH, Guest MF, Schreckenbach G, Catlow CRA, French SA, Sokol AA, Bromley ST, Thiel W, Turner AJ, Billeter S, Terstegen F, Thiel S, Kendrick J, Rogers SC, Casci J, Watson M, King F, Karlsen E, Sjovoll M, Fahmi A, Schafer A, Lennartz C (2003) Theochem 632:1 
134. Sulpizi M, Laio A, VandeVondele J, Cattaneo A, Röthlisberger U, Carloni P (2003) Proteins 52:212

135. Swart M (2003) Int J Quantum Chem 91:177

136. Tresadern G, Faulder PF, Gleeson MP, Tai Z, MacKenzie G, Burton NA, Hillier IH (2003) Theor Chem Acc 109:108

137. Vreven T, Morokuma K, Farkas O, Schlegel HB, Frisch MJ (2003) J Comput Chem 24:760

138. Vreven T, Morokuma K (2003) Theor Chem Acc 109:125

139. Yang W, Drueckhammer DG (2003) J Phys Chem B 107:5986

140. Herschend B, Baudin M, Hermansson K (2004) J Chem Phys 120:4939

141. Laio A, Gervasio FL, VandeVondele J, Sulpizi M, Röthlisberger U (2004) J Phys Chem B 108:7963

142. Pu J, Gao J, Truhlar DG (2004) J Phys Chem A 108:632

143. Riccardi D, Li GH, Cui Q (2004) J Phys Chem B 108:6467

144. Pu J, Gao J, Truhlar DG (2004) J Phys Chem A 108:5454

145. Bludsky O, Silhan M, Nachtigall P (2005) J Phys Chem B (in press)

146. Gregersen BA, York DM (2005) J Phys Chem B 109:536

147. Lin H, Truhlar DG (2005) J Phys Chem A 109:3991

148. Nam K, Gao J, York DM (2005) J Chem Theory Comput 1:2

149. Pu J, Truhlar DG (2005) Chem Phys Chem (in press)

150. Wanko M, Hoffmann M, Strodel P, Koslowski A, Thiel W, Neese F, Frauenheim T, Elstner M (2005) J Phys Chem B 109:3606

151. Zhang Y (2005) J Chem Phys 122:024114

152. Politzer P, Truhlar DG (eds) (1981) Chemical Applications of Atomic and Molecular Electrostatic Potentials Reactivity, Structure, Scattering, and Energetics of Organic, Inorganic, and Biological Systems Plenum, New York

153. Price SL, Stone AJ (1992) J Chem Soc Faraday Trans 88:1755

154. Koch U, Popelier PLA, Stone A (1995) J Chem Phys Lett 238:253

155. Matta CF, Bader RFW (2000) Proteins: Struct Func Genet 40:310

156. Minikis RM, Kairys V, Jensen JH (2001) J Phys Chem A 105:3829

157. Stillinger FH, David CW (1978) J Chem Phys 69:1473

158. Sprik M, Klein ML (1988) J Chem Phys 89:7556

159. Dick Jr BG, Overhauser AW (1958) Phy Rev 112:90

160. Van Belle D, Prevost M, Lippens G, Wodak SJ (1994) ACS Symp Ser 568:318

161. Kowall T, Foglia F, Helm L, Merbach AE (1995) J Am Chem Soc $117: 3790$

162. Banks JL, Kaminski GA, Zhou R, Mainz DT, Berne BJ, Friesner RA (1999) J Chem Phys 110:741

163. Martinez JM, Hernandez-Cobos J, Saint-Martin H, Pappalardo RR, Ortega-Blake I, Marcos ES (2000) J Chem Phys 112:2339

164. Rick SW, Stuart SJ (2002) Rev Comput Chem 18:89

165. Lamoureux G, MacKerell AD, Roux B (2003) J Chem Phys 119:5185

166. Ponder JW, Case DA (2003) Adv Protein Chem 66:27

167. Ren P, Ponder JW (2003) J Phys Chem B 107:5933

168. Kaminski GA, Stern HA, Berne BJ, Friesner RA (2004) J Phys Chem A 108:621

169. Patel S, Mackerell AD Jr, Brooks CL III (2004) J Comput Chem 25:1504

170. Rinaldi D, Rivail JL (1973) Theor Chim Acta 32:57

171. Storer JW, Giesen DJ, Hawkins GD, Lynch GC, Cramer CJ, Truhlar DG, Liotard DA (1994) ACS Symp Ser 568:24

172. Tomasi J (1994) ACS Symp Ser 1994:10

173. Cramer CJ, Truhlar Donald G (1996) Continuum solvation models In: Tapia O, Bertran J (eds) Solvents effects and chemical reactivity. Kluwer, Dordrecht, pp 1

174. Bacalis NC, Kunz AB (1985) Phys Rev B 32:4857

175. Pascual J, Pettersson LGM (1997) Chem Phys Lett 270:351

176. Stefanovich EV, Truong TN (1998) J Phys Chem B 102:3018

177. Li J, Cramer CJ, Truhlar DG (2000) Int J Quantum Chem 77:264

178. Yagüe JI, Mohammed AM, Loeffler H, Rode BM, (2001) J Phys Chem A 105:7646

179. Brooks BR, Bruccoleri RE, Olafson BD, States DJ, Swaminathan S, Karplus M (1983) J Comput Chem 4:187

180. Humbel S, Sieber S, Morokuma K (1996) J Chem Phys 105:1959

181. Waszkowycz B, Hillier IH, Gensmantel N, Payling DW (1991) J Chem Soc Perkin Trans 22025
182. Frisch MJ, Trucks GW, Schlegel HB, Scuseria GE, Robb MA, Cheeseman JR, Montgomery JJA, Vreven T, Kudin KN, Burant JC, Millam JM, Iyengar SS, Tomasi J, Barone V, Mennucci B, Cossi M, Scalmani G, Rega N, Petersson GA, Nakatsuji H, Hada M, Ehara M, Toyota K, Fukuda R, Hasegawa J, Ishida M, Nakajima T, Honda Y, Kitao O, Nakai H, Klene M, Li X, Knox JE, Hratchian HP, Cross JB, Adamo C, Jaramillo J, Gomperts R, Stratmann RE, Yazyev O, Austin AJ, Cammi R, Pomelli C, Ochterski JW, Ayala PY, Morokuma K, Voth GA, Salvador P, Dannenberg JJ, Zakrzewski VG, Dapprich S, Daniels AD, Strain MC, Farkas O, Malick DK, Rabuck AD, Raghavachari K, Foresman JB, Ortiz JV, Cui Q, Baboul AG Clifford S, Cioslowski J, Stefanov BB, Liu G, Liashenko A, Piskorz P, Komaromi I, Martin RL, Fox DJ, Keith T, Al-Laham MA, Peng CY, Nanayakkara A, Challacombe M, Gill PMW, Johnson B, Chen W, Wong MW, Gonzalez C, Pople JA (2003)Gaussian03 Gaussian, Inc: Pittsburgh PA

183. Besler BH, Merz KM Jr, Kollman PA (1990) J Comput Chem 11:431

184. Truhlar DG, Gordon MS (1990) Science 249:491

185. Morse PM (1929) Phy Rev 34:57

186. London FZ (1929) Elektrochemistry 35:552

187. Eyring H, Polanyi MZ (1931) Phys Chem B 12:279

188. Parr CA, Truhlar DG (1971) J Phys Chem 75:1844

189. Raff LM (1974) J Chem Phys 60:2220

190. Steckler R, Dykema KJ, Brown FB, Hancock GC, Truhlar DG, Valencich T (1987) J Chem Phys 87:7024

191. Jordan MJT Gilbert RG (1995) J Chem Phys 102:5669

192. Espinosa-García J, Corchado JC (1996) J Chem Phys 105:3517

193. Espinosa-García J, Corchado JC (2000) J Chem Phys 112:5731

194. Espinosa-Garcia J, Rangel C, Navarrete M, Corchado JC (2004) J Chem Phys 121:5098

195. Navarrete M, Rangel C, Espinosa-Garcia J, Corchado JC (2005) J Chem Theory Comput 1:337

196. Rangel C, Espinosa-Garcia J (2005) J Chem Phys 122:134315

197. Rangel C, Navarrete M, Espinosa-Garcia J (2005) J Phys Chem A 109:1441

198. Brenner DW (1990) Phys Rev B 42:9458

199. Brenner DW (2000) Phys Stat Sol (b) 217:23

200. Brenner DW, Shenderova OA, Harrison JA, Stuart SJ, Ni B, Sinnott SB (2002) J Phys: Condens Matter 14:782

201. Duin ACTv, Dasgupta S, Lorant F, Goddard WA (2001) J Phys Chem A 105:9396

202. Goddard WA III, Zhang Q, Uludogan M, Strachan A, Cagin T (2002) In: Cohen RE (ed) Fundamental physics of ferroelectrics 2002. American Institute of Physcis, Pasadena, vol 626, pp 45

203. Duin ACTv, Strachan A, Stewman S, Zhang Q, Xu X, Goddard WA (2003) J Phys Chem A 107:3803

204. Cheung S, Deng W-Q, van Duin ACT, Goddard WA (2005) J Phys Chem A 109:851

205. Kim Y, Corchado JC, Villa J, Xing J, Truhlar DG (2000) J Chem Phys 112:2718

206. Albu TV, Corchado JC, Truhlar DG (2001) J Phys Chem A 105:8465

207. Truhlar DG (2002) J Phys Chem A 106:5048

208. Lin H, Pu JZ, Albu TV, Truhlar DG (2004) J Phys Chem A $108: 4112$

209. Kim KH, Kim Y (2004) J Chem Phys 120:623

210. Warshel A, Weiss RM (1980) J Am Chem Soc 102:6218

211. Lin H, Truhlar DG (in press)

212. Bayly CI, Cieplak P, Cornell WD, Kollman PA (1993) J Phys Chem 97:10269

213. Laio A, VandeVondele J, Rothlisberger U (2002) J Phys Chem B 106

214. Storer JW, Giesen DJ, Cramer CJ, Truhlar DG (1995) J ComputAid Mol Des 9:87

215. Li J, Zhu T, Cramer CJ, Truhlar DG (1998) J Phys Chem A $102: 1820$

216. Li J, Williams B, Cramer CJ, Truhlar DG (1999) J Chem Phys 110:724

217. Winget P, Thompson JD, Xidos JD, Cramer CJ, Truhlar DG (2002) J Phys Chem A 106:10707 
218. Brom JM, Schmitz BJ, Thompson JD, Cramer CJ, Truhlar DG, (2003) J Phys Chem A 107:6483

219. Thompson JD, Cramer CJ, Truhlar DG (2003) J Comput Chem 24:1291

220. Kelly C, Cramer CJ, Truhlar Donald G (in press)

221. Mortier WJ, Ghosh SK, Shankar S (1986) J Am Chem Soc 108:4315

222. Rappé AK, Goddard WA (1991) J Phys Chem 95:3358

223. Kitao O, Ogawa T (2003) Mol Phys 101:3
224. Ogawa T, Kurita N, Sekino H, Kitao O, Tanaka S (2003) Chem Phys Lett 374:271

225. Ogawa T, Kurita N, Sekino H, Kitao O, Tanaka S (2004) Chem Phys Lett 397:382

226. Gogonea V, Merz KM Jr (2000) J Phys Chem B 104:2117

227. Ackland GJ, Finnis MW, Vitek V (1988) J Phys F: Met Phys 18:L153

228. Jasper AW, Schultz NE, Truhlar DG (2005) J Phys Chem B 109:3915 